\title{
The Anatomy of the Dog Soft Palate. III. Histological Evaluation of the Caudal Soft Palate in Brachycephalic Neonates
}

\author{
MICHELA PICHETTO,${ }^{1}$ SILVANA ARRIGHI, ${ }^{2 *}$ MATTEO GOBBETTI, ${ }^{1}$ \\ AND STEFANO ROMUSSI ${ }^{1}$ \\ ${ }^{1}$ Department of Veterinary Science and Public Health (DIVET), Faculty of Veterinary \\ Medicine, Università degli Studi di Milano, Italy \\ ${ }^{2}$ Department of Health, Animal Science and Food Safety (VESPA), Laboratory of Anatomy \\ and Confocal Microscopy. Faculty of Veterinary Medicine, \\ Università degli Studi di Milano, Italy
}

\begin{abstract}
A thickened and abnormally long soft palate is mostly involved in the pathogenesis of both nasopharyngeal and oropharyngeal narrowing, affecting the respiratory activity in virtually all of the brachycephalic dogs suffering from Brachycephalic Airway Obstructive Syndrome (BAOS). The morphology of the soft palate in adult mesaticephalic and brachycephalic dogs has been previously described. In this article specimens from brachycephalic dog neonates $(N=10)$ dead from one to $3 \mathrm{hr}$ after birth of unrelated conditions were collected and histologically evaluated at three transverse levels to describe the microscopic aspect of the caudal part of the soft palate. The soft palate of neonate brachycephalic dogs was histologically characterized by a musculoconnective axis containing salivary glands and coated by a mucosal layer on both the nasopharyngeal and the oral side. Quali-quantitative features, such as thickened superficial epithelium, broad oedema of the lamina propria, mucous gland hyperplasia and diverse muscular modifications described in adult brachycephalic dogs, were not observed in the soft palate of brachycephalic neonate dogs. The lack of tissue lesions in the soft palate of newborn brachycephalic dogs further supports the hypothesis that inspiratory depression during the inspiratory phase causes chronic vibration and microtrauma, which lead to soft palate alterations in adult brachycephalic dogs starting from the earliest grade of the respiratory syndrome. Overall, this study provides baseline information for the comprehension of the pathogenesis of BAOS. Anat Rec, 298:618-623, 2015. 으 2014 Wiley Periodicals, Inc.
\end{abstract}

Key words: soft palate; brachycephalic dog; histology; neonates; brachycephalic airway obstructive syndrome (BAOS)

\section{INTRODUCTION}

The "thick, redundant and hyperplastic" morphology of the soft palate is widely recognized as one of the main morphological alterations contributing to the pathogenesis of the canine brachycephalic airway obstructive syndrome (BAOS) (Aron and Crowe, 1985; Hendricks et al., 1987; Wykes, 1991; Hendricks, 1992; Findji and Duprè, 2008). Recent studies demonstrated that soft palate oversizing is the most common finding in patients with BAOS, occurring in $85-100 \%$ of cases (Lorinson et al., 1997; Poncet et al., 2005, 2006; Torrez
Michela Pichetto is currently affiliated with Clinica Veterinaria, Dr. Boldini - 70, Via F. Agello, I-25015 Desenzano Del Garda (BS), Italy

Grant sponsor: Università degli Studi di Milano.

*Correspondence to: Silvana Arrighi, Department of Health, Animal Science and Food Safety, Laboratory of Anatomy and Confocal Microscopy, 2, Via Trentacoste, I-20134 Milano, Italy. Fax: +39.0250315746. E-mail: silvana.arrighi@unimi.it

Received 28 May 2014; Accepted 10 August 2014.

DOI 10.1002/ar.23054

Published online 25 September 2014 in Wiley Online Library (wileyonlinelibrary.com). 
and Hunt, 2006; Riecks et al., 2007; Fasanella et al., 2010).

A previous study shows that the distal part of the soft palate of adult brachycephalic dogs possesses peculiar features (Pichetto et al., 2011), which on the whole can account for its increased thickness. Histological peculiarities, such as thickened mucosal epithelia, general oedema of the connective tissue, glandular hyperplasia and many different muscular modifications were commonly described (Pichetto et al., 2011). On the other hand, such histological features could not be found in the corresponding part of the soft palate of mesaticephalic dog breeds as previously described in a related article (Arrighi et al., 2011).

Although there is evidence that the skull bone shortening typical of brachycephalic dogs does not occur together with a diminished development of soft tissues (Harvey, 1989), in our previous studies we could not discern whether part of the augmented thickness of the soft palate was due to an inherited defect or instead was acquired after chronic barotraumas during respiration, as previously hypothesized by other authors (Hendricks, 2004).

Therefore, this study was aimed at describing the microanatomy of the soft palate in newborn brachycephalic dogs under the hypothesis that the histological alterations found in adult brachycephalic dogs might not be present in neonates.

This article constitutes the final part of three companion papers (Arrighi et al., 2011; Pichetto et al., 2011) which were aimed at giving a wide anatomical description in different canine head shapes.

\section{MATERIALS AND METHODS}

Samples were collected from neonate English bulldogs $(N=10)$ spontaneously dead from 1 to $3 \mathrm{hr}$ after birth of unrelated conditions.

The dogs' heads were fixed in toto in $10 \%$ neutral formalin for 20-30 days, then the distal part of the soft palate was dissected along a curved line crossing transversally from the caudal border of the fossa tonsillaris to the same point on the opposite side, trying to mimic the surgical dissection performed in the BAOS affected animals (Hobson, 1995; Pichetto et al., 2011).

Afterwards, the specimens were processed for histology and embedded in paraffin. Each block was examined at three transverse levels starting cranially from the dissection line and proceeding aborally with serial sections.

Microscopic evaluation of histological and histochemical features was performed. For this purpose, serial sections were stained as described in Arrighi et al. (2011). Briefly, Haematoxylin and Eosin technique (H\&E), Mallory trichromic stain, sequential Alcian Blue $\mathrm{pH}$ 2.5/Periodic Acid-Schiff (AB/PAS) staining technique and High Iron Diamine/Alcian Blue 8GX pH 2.5 (HID/AB) reactions were applied to describe the major tissue types constituting the soft palate and to characterize the mucin types within glandular tissue.

\section{RESULTS}

In Haematoxylin and Eosin (H\&E)-stained sections, the caudal part of the soft palate in neonate brachycephalic dogs was characteristically organized into a main deep musculo-connective axis containing salivary glands, coated by a folded mucosal lining on both the nasopharyngeal and oral side (Fig. 1a). Constant and regular changes were observed in the neonatal soft palate morphology from the more oral sections (Level 1) and proceeding aborally (Levels 2 and 3).

At Level 1, the nasopharyngeal side was lined by a ciliated pseudostratified epithelium with mucous cells (Fig. 1a,b), anchored by a straight basement membrane to the loose connective tissue underneath. A vascular plexus, mainly formed by small capillaries, was present in the outermost connective tissue of the lamina propria together with widespread lymphocytic infiltrate. The oral side was lined by a pluristratified squamous epithelium (Fig. 1c) supported by a wavy basement membrane organized into irregular finger-like papillae. At Level 2 the pseudostratified epithelium lining the nasal side was substituted by a pluristratified squamous epithelium, starting from the lateral sides. At Level 3 both nasopharyngeal and oral sides were covered by similar epithelia.

The palatine musculature formed the most prominent axial feature of the organ (Fig. 1a), composed by the coupled longitudinally oriented musculus palatinus and by the paired, laterally arranged muscles, mostly converging toward the dorsal side (Fig. 1d). The latter were most likely the tensor and the levator veli palatini in the more rostral sections, and the palatopharyngeus aborally (Evans, 1993). Palatine muscles were made up by hypotrophic, small and homogeneously sized fibers having an immature aspect, and numerous satellite cells (Figs. 1e and $2 \mathrm{a}$ ). The aponeurosis palatina was roughly outlined at Levels 1 or 2 , according to the subject. It was never neat in any of the specimens examined.

Palatine salivary glands were specially crowded at the oral side, where they extended all over the palate width. At the nasopharyngeal side the glands were placed laterally (Fig. 1a). Glandular lobules were intermingled with the palatine musculature and their excretory ducts opened onto the mucosal surface. At both sides, palatine glands were composed of mixed acini, in which mucous cells were preponderant and serous cells formed demilunes around the mucous cells (Fig. 2a,b). Excretory ducts were lined by an isoprismatic bistratified epithelium devoid of morphological evidence of secretory activity (Figs. 1b,c and 2a,b).

Histochemical reactivity of the nasopharyngeal epithelium showed mixed Alcian- and PAS-positivity of the mucous cells, the most part of which were also HID-positive (Fig. 2e). A marked PAS-positivity was found in the oral epithelium at the level of all cell layers except for the basal one, which was devoid of any AB/PAS reactivity (Fig. 2f).

The histochemical affinity of the palatine glands was homogeneous, independent of their localization. The mucous cells showed separate Alcian- and PAS-positivity, or sometimes a mixed reactivity with prevalent alcianophily (Fig. 2c). By HID/AB reaction, the mucous elements were mostly HID-positive (Fig. 2d). Glandular serous cells contained PAS-positive granules.

The epithelial covering of the excretory ducts was unreactive, but contained an alcianophil intralumenal secretory matter, which was also detected in sporadic intraepithelial cavities ("cysts").

\section{DISCUSSION}

Brachycephalia is due to a local chondrodysplasia, which occurs as a result of domestication. Early 

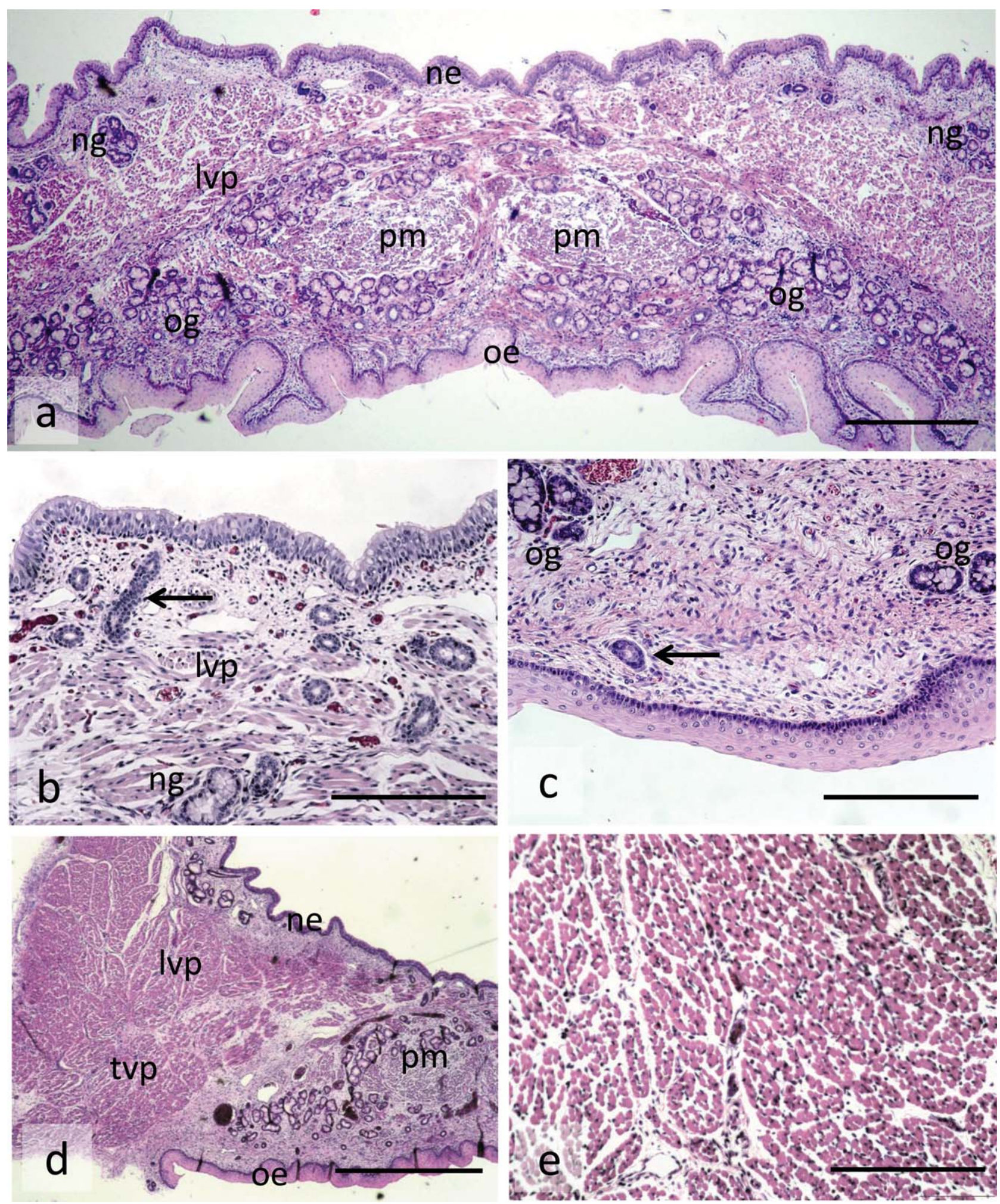

Fig. 1. Soft palate morphology in brachycephalic neonate dogs. Level 1 histologic sections are shown. (a) At low magnification the soft palate stratigraphy can be seen, from the dorsal, nasopharyngeal side (top) to the ventral, oral side (bottom). (b) The nasal mucosa is characterized by columnar pseudostratified ciliated epithelium containing mucous cells. In the outermost connective tissue of the lamina propria a vascular plexus is present. Glandular acini can be seen (ng), together with excretory ducts opening into the mucosal surface (arrow). (c) Pluristratified squamous epithelium

lines the oral mucosa, accompanied by an underlying loose connective tissue. Salivary glands can also be seen (og). Arrow indicates an excretory duct. (d) Laterally, the soft palate is reached by skeletal muscles, which converge mostly toward the dorsal side. (e) Palatine muscles are made up by small and regular fibers. ne, nasopharyngeal epithelium, $\mathbf{n g}$, nasal glands, pm, m. palatinus, og, oral glands, oe, oral epithelium, Ivp, $m$. levator veli palatini, tvp, $m$. tensor veli palatini. H\&E. Scale bars: $\mathrm{a}=500 \mu \mathrm{m} ; \mathrm{b}, \mathrm{c}, \mathrm{e}=200 \mu \mathrm{m}$; $\mathrm{d}=1000 \mu \mathrm{m}$. 

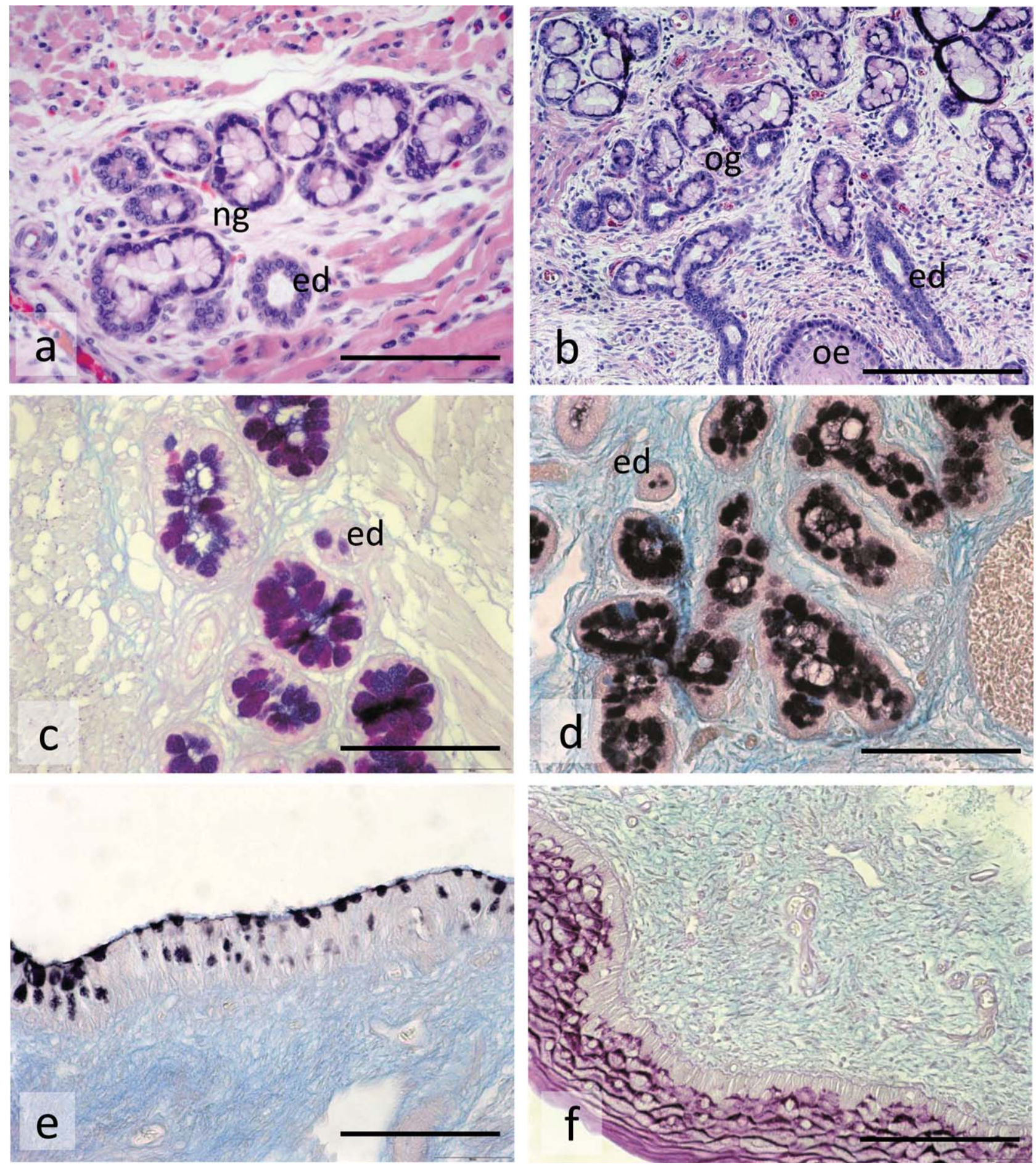

Fig. 2. Palatine salivary glands of the nasopharyngeal (a) and oral (b) sides. At the oral side the glands are more numerous and crowded. At both sides the glandular lobules are composed of mixed acini, in which mucous cells are preponderant and serous cells form demilunes around the mucous cells. H\&E. (c) By Alcian/PAS reaction, the gland mucous cells show a mixed Alcian- and PAS-positivity. (d) By HID/AB reaction,

the gland mucous cells are mostly HID-positive. (e) By HID/AB reaction, the majority of the mucous cells of the nasopharyngeal epithelium are HID-positive. (f) By Alcian/PAS reaction a marked PAS-positivity is shown in the oral epithelium at the level of all cell layers except for the basal one. ng, nasal glands, og, oral glands, ed, excretory duct, oe, oral epithelium. Scale bars: a,c,d,e,f $=100 \mu \mathrm{m} ; \mathrm{b}=200 \mu \mathrm{m}$. 
synostosis occurs in the cartilage of the base of the skull, leading to a shortened longitudinal axis of the skull (Hennet and Harvey, 1992).

Brachycephalic dogs and cats are very popular in many parts of the world for their child-like characteristics, which instinctively attract most people. Unfortunately, the brachycephalic conformation of the skull results in a high incidence of associated diseases, mainly of respiratory nature, which are designated as Brachycephalic Airway Obstructive Syndrome (BAOS) (Koch et al., 2003).

Soft palate alterations have been suggested as a principal component of BAOS. Its increased volume is one of the key elements contributing to the narrowing of the nasopharyngeal lumen during nasal air flow recruitment and of the oropharyngeal lumen during open mouth breathing.

It is widely accepted that the discordant development between bone and soft tissue of the skull results in a relatively longer soft palate (Harvey, 1989). On the other hand, it is not clear if this aspect could somehow also influence the increased thickness of the soft palate.

We found in our previous study on adult brachycephalic dogs that many different microscopic changes affected soft palate tissues and we hypothesized that these modifications could have been responsible for the increased thickness of the organ (Pichetto et al., 2011). The most important microscopic observations were signs of hyperplasia and intracellular oedema of mucosal epithelia, pervasive oedema of the connective tissue, glandular hyperplasia/mucocele and many muscular abnormalities. Surprisingly, the soft palate of all dogs in the brachycephalic group classified as Grade I BAOS patients were affected by similar alterations. Therefore, this study focused on the histological evaluation of the soft palate in newborn brachycephalic individuals, in an attempt to investigate whether at least part of the augmented thickness of the soft palate might be due to acquired events. Only the distal part of the soft palate was evaluated in neonate brachycephalic dogs of this study, in such a way to allow an exact comparison with the portion of the palate resected during surgery in the BAOS affected adults examined in the companion paper II (Pichetto et al., 2011).

None of the layers constituting the soft palate of neonate brachycephalic dogs showed signs of oedema or other histopathologic alterations that are highly typical of the soft palate morphology in adult brachycephalic dogs.

Thus, it is tempting to speculate that these changes occur during postnatal life and might be the result of airway turbulence and consequent chronic barotraumas, as previously hypothesized (Hendricks, 2004).

Additional morphometric studies, in which ratios between skull and palate measurements were compared at different ages, are needed to definitely quantify the impact of both inherited discordant development of the skull or such acquired alterations.

The microscopical results obtained highlight a well organized multilayered organ, made up by a musculoconnective axis containing also salivary glands and covered by a mucosa, differently structured at the nasopharyngeal and oral sides.

The different aspect of the mucosal layer at the two dorsal and ventral sides, together with the different organization at the boundary between epithelial tissue and the supporting connective, might sustain different functional performances at the two mucosal sides, as underlined in the mesaticephalic dog soft palate (Arrighi et al., 2011) and previously suggested by Paulsen and Thale (1998) in the human soft palate.

The density of the salivary glands, particularly at the oral side, together with their histochemical reactivity, supports the hypothesis that moisture and lubrication of the superficial mucosal layer is crucial to prevent injuries caused by friction during food intake (Kuehn and Moon, 2005). AB/PAS and HID/AB stains demonstrated a prevalent contribution of sulphated mucin to the saliva, which is known to make the mucous secretion denser. A similar reactivity was shown by the mucous cells of the nasopharyngeal mucosa covering the more rostral soft palate sections. It is well-known that these cells are part of the mucociliary apparatus, providing the mucus blanket which lines and protects nasal airways against injury at the level of the upper respiratory airways (Voynow and Rubin, 2009).

The palatine musculature observed in neonate soft palates is built by muscles localized in the longitudinal axis, the paired $m$. palatinus (equivalent of the human uvula), and others arising from adjacent bone structures and fanning out in the centre of the soft palate, the tensor and the levator veli palatini in the more rostral sections, and the $m$. palatopharyngeus aborally. The palatine aponeurosis is not well developed yet. The structural aspect of the palatine muscles is immature, with hypotrophic, small and regular fibers, and many satellite cells.

As reported in a previous companion paper severe muscular lesions were detected in a series of adult brachycephalic dogs: heterogeneous fibre size variations (atrophy-hypertrophy), hyaline degeneration, Zenker necrosis, endomysial and perimysial fibrosis, impossibility to identify palatine muscle due to severe muscle disorganization (Pichetto et al., 2011). None of these extensive degenerative muscular lesions detected in adult brachycephalic dogs were found in the neonate dogs. Therefore, we can speculate that these severe alterations are acquired and might be due to chronic barotrauma. A plausible hypothesis is that these myopathic changes may ultimately impair the ability of muscles to maintain pharyngeal patency. This condition synergistically promotes upper airway obstruction progress and thus plays an important role in the pathogenesis of BAOS (Petrof et al., 1996).

In conclusion, our study reveals the microanatomical features of the soft palate of neonate English bulldogs. Our results confirm the initial hypothesis that histological alterations found in adult brachycephalic dogs are not present at birth and that the soft palate becomes progressively thicker during postnatal life due to dysfunctional inspiratory efforts and consequent chronic barotrauma.

The present study does not allow us to draw definitive conclusions about the inherited or acquired nature of soft palate hyperplasia, especially with regard to its increased length. For this purpose, further morphometric studies, comparing measurements of soft and hard tissues of the skull of brachycephalic dogs at different stages of growth, are needed.

Overall, we do believe that the present and the companion articles on the mesaticephalic and brachycephalic dog soft palate, respectively, might represent a comprehensive landmark study to which both anatomists, 
pathologists and clinicians could refer. Moreover, it can be speculated that knowledge of the detailed microanatomy of soft palate tissues might be of some help to get deeper insights into the pathogenesis of BAOS in brachycephalic breeds.

\section{ACKNOWLEDGEMENTS}

The authors would like to thank the English bulldog breeders, who kindly collaborated in the collection of the dog soft palates used in this research. Mr. Paolo Stortini (VESPA, Università degli Studi di Milano) is gratefully acknowledged for his experienced and skilful technical support.

Part of this work was presented at the XXVIII Congress of the European Association of Veterinary Anatomists (EAVA), Paris, France, July 28-31, 2010 (Proceedings in Anat. Histol. Embryol., 39/4, 263-264, 2010).

\section{LITERATURE CITED}

Aron DN, Crowe DT. 1985. Upper airway obstruction. Vet Clin North Am Small Anim Pract 15:902-904.

Arrighi S, Pichetto M, Roccabianca P, Romussi S. 2011. The anatomy of the dog soft palate. I. Histological evaluation in mesaticephalic breeds. Anat Rec 294:1261-1266.

Evans HE. 1993. Miller's anatomy of the dog. 3rd ed. Philadelphia: Saunders.

Fasanella FJ, Shivley JM, Wardlaw JL, Givaruangsawat S. 2010 Brachycephalic airway obstructive syndrome in dogs: 90 cases (1991-2008). J Am Vet Med Assoc 237:1048-1051.

Findji L, Duprè G. 2008. Folded flap palatoplasty for treatment of elongated soft palates in 55 dogs. Vet Med Austria 95:56-63.

Harvey CE. 1989. Inherited and congenital airway conditions. J Small Anim Pract 30:184-187.

Hendricks JC. 1992. Brachycephalic airway syndrome. Vet Clin North Am Small Anim Pract 22:1145-1153.

Hendricks JC. 2004. Brachycephalic airway syndrome. In: King LG, editor. Textbook of respiratory disease in dogs and cats. St Louis: Saunders. p 310-318.
Hendricks JC, Kline LR, Kovalski RJ, O'Brien JA, Morrison AR, Pack AI. 1987. The English Bulldog: a natural model of sleepdisordered breathing. J Appl Physiol 63:1344-1350.

Hennet PR, Harvey CE. 1992. Craniofacial development and growth in the dog. J Vet Dent 9:11-18.

Hobson HP. 1995. Brachycephalic syndrome. Seminars in Veterinary Medicine. Small Anim Surg 10:109-114.

Koch DA, Arnold S, Huber M, Mantovam PM. 2003. Brachycephalic syndrome in dogs. Compend Contin Educ Pract Vet 25:48-55.

Kuehn DP, Moon JB. 2005. Histologic study of intravelar structures in normal human adult specimens. Cleft Palate Craniofac J 42: 481-489.

Lorinson D, Bright R, White RAS. 1997. Brachycephalic airway obstruction syndrome-a review of 118 cases. Can Pract 22:18-21.

Paulsen F, Thale A. 1998. Epithelial-connective tissue boundary in the oral part of the human soft palate. J Anat 193:457-467.

Petrof BJ, Hendricks JC, Pack AL. 1996. Does upper airway muscle injury trigger a vicious cycle in obstructive sleep apnea? A hypothesis. Sleep 19:465-471.

Pichetto M, Arrighi S, Roccabianca P, Romussi S. 2011. The anatomy of the dog soft palate. II. Histological evaluation of the caudal soft palate in brachycephalic breeds with grade 1 brachycephalic airway obstructive syndrome (BAOS). Anat Rec 294: 1267-1272.

Poncet CM, Dupré GP, Freiche VG, Estrada MM, Poubanne YA, Bouvy BM. 2005. Prevalence of gastrointestinal tract lesions in 73 brachycephalic dogs with upper respiratory syndrome. J Small Anim Pract 46:273-279.

Poncet CM, Dupré GP, Freiche VG, Bouvy BM. 2006. Long-term results of upper respiratory syndrome surgery and gastrointestinal tract medical treatment in 51 brachycephalic dogs. J Small Anim Pract 47:137-142.

Riecks TW, Birchard SJ, Stephens JA. 2007. Surgical correction of brachycephalic syndrome in dogs: 62 cases (1991-2004). J Am Vet Med Assoc 230:1324-1328.

Torrez CV, Hunt GB. 2006. Results of surgical correction of abnormalities associated with brachycephalic airway obstruction syndrome in dogs in Australia. J Small Anim Pract 47:150-154.

Voynow JA, Rubin BK. 2009. Mucins, mucus, and sputum. Chest 135:505-512.

Wykes PM. 1991. Brachycephalic airway obstructive syndrome. Probl Vet Med 3:188-197. 